\title{
Social scaling of extrapersonal space: Target objects are judged as closer when the reference frame is a human agent with available movement potentialities
}

\author{
C. Fini ${ }^{\mathrm{a}, \mathrm{b}, *}$, M. Brass ${ }^{\mathrm{c}}, \mathrm{G} \cdot$ Committeri $^{\mathrm{a}, \mathrm{b}}$ \\ ${ }^{a}$ Laboratory of Neuropsychology and Cognitive Neuroscience, Department of Neuroscience, Imaging and Clinical Sciences, University G. d'Annunzio, \\ via dei Vestini 33, 66100 Chieti Scalo, Italy \\ ${ }^{\mathrm{b}}$ ITAB, Foundation G. d'Annunzio, via dei Vestini 33, 66100 Chieti Scalo, Italy \\ ${ }^{\mathrm{c}}$ Department of Experimental Psychology, Ghent University, Henri-Dunantlaan 2, 9000 Ghent, Belgium
}

\section{A R T I C L E I N F O}

\section{Article history:}

Received 5 November 2013

Revised 28 July 2014

Accepted 23 August 2014

Available online 17 October 2014

\section{Keywords:}

Reference frames

Allocentric

Body

Motor potentialities

Virtual reality

Ecological validity

\begin{abstract}
A B S T R A C T
Space perception depends on our motion potentialities and our intended actions are affected by space perception. Research on peripersonal space (the space in reaching distance) shows that we perceive an object as being closer when we (Witt, Proffitt, \& Epstein, 2005; Witt \& Proffitt, 2008) or another actor (Costantini, Ambrosini, Sinigaglia, \& Gallese, 2011; Bloesch, Davoli, Roth, Brockmole, \& Abrams, 2012) can interact with it. Similarly, an object only triggers specific movements when it is placed in our peripersonal space (Costantini, Ambrosini, Tieri, Sinigaglia, \& Committeri, 2010) or in the other's peripersonal space (Costantini, Committeri, \& Sinigaglia, 2011; Cardellicchio, Sinigaglia, \& Costantini, 2013). Moreover, also the extrapersonal space (the space outside reaching distance) seems to be perceived in relation to our movement capabilities: the more effort it takes to cover a distance, the greater we perceive the distance to be (Proffitt, Stefanucci, Banton, \& Epstein, 2003; Sugovic \& Witt, 2013). However, not much is known about the influence of the other's movement potentialities on our extrapersonal space perception. Three experiments were carried out investigating the categorization of distance in extrapersonal space using human or non-human allocentric reference frames (RF). Subjects were asked to judge the distance ("Near" or "Far") of a target object (a beach umbrella) placed at progressively increasing or decreasing distances until a change from near to far or vice versa was reported. In the first experiment we found a significant "Near space extension" when the allocentric RF was a human virtual agent instead of a static, inanimate object. In the second experiment we tested whether the "Near space extension" depended on the anatomical structure of the RF or its movement potentialities by adding a wooden dummy. The "Near space extension" was only observed for the human agent but not for the dummy. Finally, to rule out the possibility that the effect was simply due to a line-of-sight mechanism (visual perspective taking) we compared the human agent free to move with the same agent tied to a pole with a rope, thus reducing movement potentialities while maintaining equal visual accessibility. The "Near space extension" disappeared when this manipulation was introduced, showing that movement potentialities are the relevant factor for such an effect. Our results demonstrate for the first time that during allocentric distance judgments within extrapersonal space, we implicitly process the movement potentialities of the RF. A target object is perceived as being closer when the allocentric RF is a human with
\end{abstract}

\footnotetext{
* Corresponding author at: Laboratory of Neuropsychology and Cognitive Neuroscience, Department of Neuroscience, Imaging and Clinical Sciences, University G. d'Annunzio, via dei Vestini 33, 66100 Chieti Scalo, Italy. Tel.: +39 0871 3556945; fax: +39 08713556930.

E-mail addresses: chiara.fini@unich.it (C. Fini), marcel.brass@ugent.be (M. Brass), gcommitteri@unich.it (G. Committeri).
} 
available movement potentialities, suggesting a mechanism of social scaling of extrapersonal space processing.

ㄷ 2014 Published by Elsevier B.V.

\section{Introduction}

The way we perceive the space around us strongly depends on our action potentialities. We perceive a target at a shorter distance, for example, when we hold a tool with the intention to reach it (Witt, Proffitt, \& Epstein, 2005; Witt \& Proffitt, 2008).

The link between action potentialities and space perception also extends to the space outside reaching distance (extrapersonal space) (Grusser, 1983; Cutting \& Vishton, 1995; Rizzolatti, Fadiga, Fogassi \& Gallese, 1997; Previc, 1998). Extrapersonal space seems indeed to be categorized not only in relation to relevant optical and ocular-motor variables, but also as a function of a person's current potentialities to perform intended actions (Witt, Proffitt, \& Epstein, 2004). As a matter of fact, a distance appears greater when the effort associated with walking increases, for instance when subjects are carrying a heavy backpack (Proffitt, Stefanucci, Banton, \& Epstein, 2003) or when they are old (Sugovic \& Witt, 2013). Therefore, the potentialities of our body to achieve behavioral goals strongly influences our distance judgments (Proffitt, 2006).

This raises the question whether we also consider the movement potentialities of others when we judge space in relation to them. Previous research indicates that the action opportunities of other agents in the visual scene induces specific motor acts in the observer, showing a "remapping" of the observer into the other's reaching space (Costantini, Committeri, \& Sinigaglia, 2011; Cardellicchio, Sinigaglia, \& Costantini, 2013). Such an effect disappears when a transparent barrier between the actor and the target of his action is introduced. Moreover, observing someone else using a tool to reach a target, while we ourselves are holding a tool, results in an underestimation of the target distance (Costantini, Ambrosini, Sinigaglia, \& Gallese, 2011). These findings strongly suggest that we construct a spatial representation considering our own and other's action opportunities by matching our own with other's reaching space. Whether such a remapping of space induced by our own or other people's action potentialities also holds true for extrapersonal space, is still an open question.

We know that people tend to automatically adopt the other person's visuospatial perspective (see Tversky \& Hard, 2009; Samson, Apperly, Braithwaite, Andrews, \& Scott, 2010; Surtees \& Apperly, 2012). Even in the absence of communication, the mere presence of another person in the position to act on some objects has been shown to induce a description of spatial relations from that person's point of view (Tversky \& Hard, 2009). These results show that when confronted with others, people may naturally take their perspective and perceive the world from their eyes and from the position of their bodies. It has been demonstrated that people actually disengage from an egocentric reference frame (RF) when they represent the scene from the perspective of another person, with an "altercentric" remapping of space, i.e. remapping of objects and locations with reference to the other person's body (Becchio, Del Giudice, Latini-Corazzini, \& Pia, 2011).

The aim of the present study was to investigate whether the other's body, with its action potentialities, constitutes a particular kind of allocentric RF for extrapersonal space categorization. We took advantage of virtual reality (VR) in order to manipulate the nature of the adopted RF during a distance categorization task. Participants were required to carry out "Near/Far" judgments through which we calculated a spatial threshold using the psychophysical limits method.

We showed that adopting another human's body as RF leads to an extension of the sector of extrapersonal space judged as "Near" when compared to a static object as RF (Exp. 1). Then we demonstrated that to induce that effect, the RF has to be processed with a biological apparatus (Exp. 2), and as able to spend his movement potentialities (Exp. 3).

\section{Experiment 1}

The aim of the first experiment was to explore whether adopting an Other-centered compared to an Object-centered RF could influence a person's extrapersonal space categorization. Our expectations entailed that, during the "Near/Far" judgment of the target location in extrapersonal space, there would be a "Near space extension" when adopting as RF a human agent vs. an object, because of the implicit processing of human movement potentialities.

\subsection{Materials and methods}

Twenty-three healthy subjects took part in this experiment (16 females, mean age 25 years, range 20-29). All subjects were right-handed, had normal or corrected-tonormal visual acuity and were naïve as to the purposes of the experiment. The study was approved by the Ethics Committee of the "G. d'Annunzio" University in Chieti, Italy, and conducted in accordance with the ethical standards of the 1964 Declaration of Helsinki.

Stimuli included a 3D scene created by means of a 3D modelling software (3D Studio Max 4.2, Autodesk, Discreet). The scene was a 3D environment, representing a square arena defined by the two short lateral wings and the long central wing of a palace (Fig. 1). In the first set of stimuli (Fig. 1A) a green beach umbrella (Object RF) was located $45^{\circ}$ to the right (left) of the central camera representing the participant's perspective, and a target red beach umbrella was located along a central vector aligned with the Object RF at 27 different distances (from $2 \mathrm{~m}$ to $54 \mathrm{~m}$ ). The second set of stimuli (Fig. 1B) was 


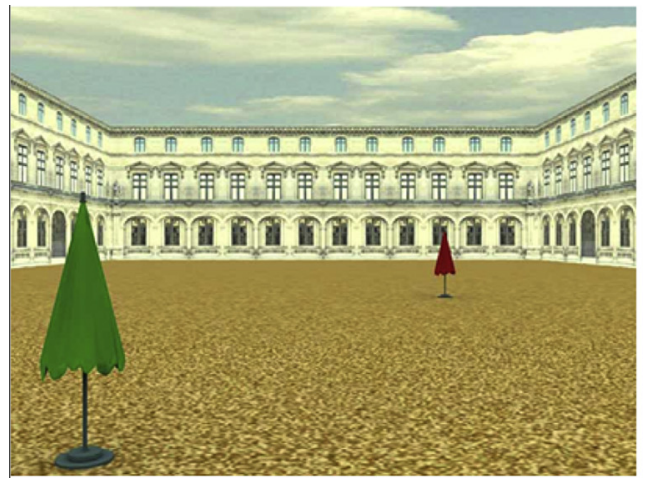

(A) Object RF

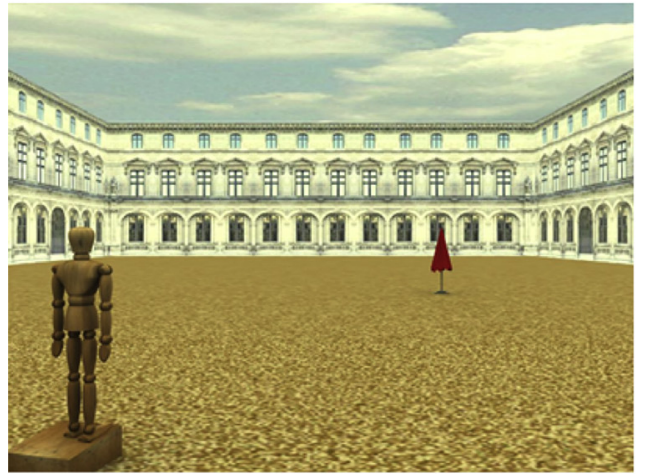

(C) Dummy RF

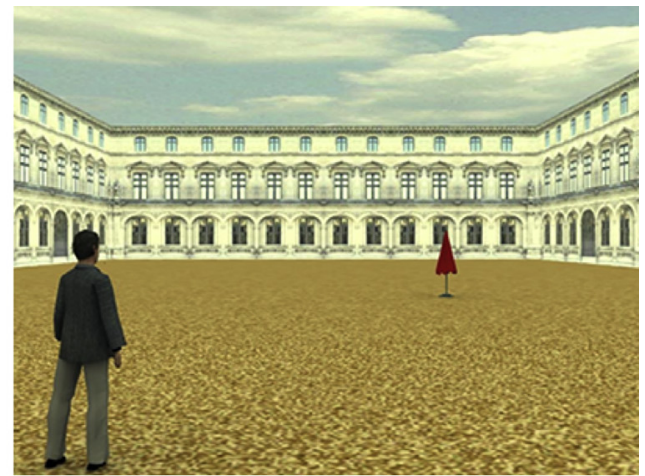

(B) Other RF

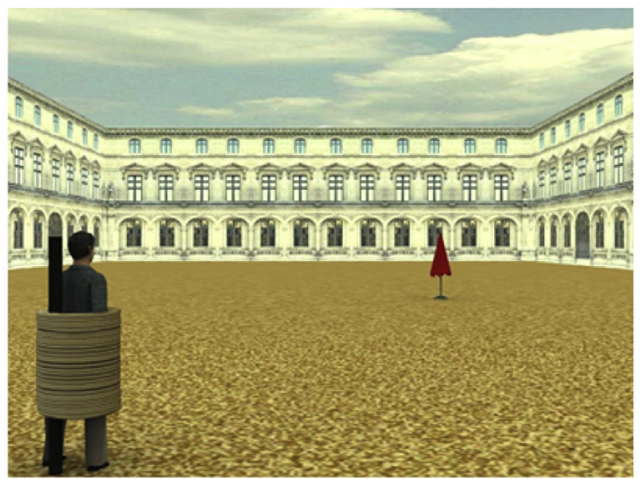

(D) Tied-Other RF

Fig. 1. Stimuli in 3D scenario used in the three experiments: Stimuli (A and B) were used in Experiment 1 together with the egocentric, Self condition (not shown); stimuli $(A-C)$ were used in Experiment 2; stimuli (A, B and D) were used in Experiment 3.

identical to the first one, except for the presence of a virtual man or avatar (Other RF) instead of the green umbrella. The avatar and the umbrella were $177 \mathrm{~cm}$ and $192 \mathrm{~cm}$ tall, respectively, resembling their ecological relative proportion in a real scenario. Note also that the avatar and the umbrella had the same spatial extension in the anterior direction. In a third set of stimuli (not shown), only the red target umbrella was present on the scene, along a central vector aligned to the central camera (Self $\mathrm{RF}$ ). The last, egocentric condition was included to make the distance categorization task more ecological, given that real-world spatial computations in extrapersonal space are characterized by a continuous shift between egocentric and allocentric RFs.

The stimuli were administered through the limit method. This is a method for measuring perceptive thresholds, in which the subject is presented with series of stimuli with progressively increasing or decreasing (in steps of a predetermined value) intensity (distance in our case), until he/she reports to feel a change. Each experimental series started with a white fixation cross $\left(1.5^{\circ} \times 1.5^{\circ}\right)$ on a black background $(2500 \mathrm{~ms})$ and consisted of 27 potential trials in which the red beach umbrella was located at 27 different distances from the reference frame (RF). Each trial lasted $2500 \mathrm{~ms}$ and was followed by a white fixation cross on a black background for $2500 \mathrm{~ms}$. Subjects were asked to categorize the red beach umbrella as "Near"
("Vicino") or "Far" ("Lontano") from the two different RFs, by pressing two different buttons arranged horizontally on the computer keyboard and counterbalanced in the "Near"/"Far" judgment. The "Near"/"Far" judgments were requested to be immediate and subjective, and had to be expressed while the image was shown on the screen. In the ascending series, the red umbrella was progressively moved away from the RF until the participants provided three consecutive "Far" judgments. In the descending series, the red umbrella was progressively moved closer to the RF until the participants provided three consecutive "Near" judgments. This was done to ensure judgment consistency. The point where participants expressed a transition from "Far" to "Near" (descending series) and from "Near" to "Far" (ascending series), was called Judgment's transition threshold (JTT). A mean JTT was calculated for each subject. Series were averaged together to obtain a final mean JTT referring to the different RFs. Higher JTT values show a categorization of space as "Near" at longer target distance compared to lower JTT values. In other words, the higher the JTT, the broader the space categorized as "Near". Each series was repeated 4 times for each RF. Each subject was thus submitted to 24 randomized experimental series ( 3 RFs: Self, Other, Object $\times 8$ series type: 4 ascending, 4 descending). Stimuli were presented at full screen on a $17^{\prime}$ computer display placed at a distance of $57 \mathrm{~cm}$ in front of the subject. The presentation of the 
stimuli and the recording of the participant's responses were controlled by a custom software (Gaglab, developed by Gaspare Galati at the Department of Psychology, Sapienza Università di Roma, Italy), implemented in MATLAB (the MathWorks Inc., Natick, MA, USA) using Cogent Graphics (developed by John Romaya at the LON, Wellcome Department of Imaging Neuroscience, UCL, London UK).

\subsection{Results and discussion}

A repeated measures analysis of variance (ANOVA) comparing JTT in the three RF conditions (Self, Other, Object) yielded a significant RF-based distance categorization effect $\left(F_{(2,44)}=72.4, p<0.001, \eta^{2}=0.8\right)$. Post-hoc tests (Newman Keuls) showed a significantly higher JTT in the Self $(J T T=13.40 \mathrm{~m}, \mathrm{SD}=3.06)$ respect to both the Other $(\mathrm{JTT}=10.78 \mathrm{~m}, \mathrm{SD}=3.15 ; p<0.001)$ and the Object $(\mathrm{JTT}=10.08 \mathrm{~m}, \mathrm{SD}=3.15 ; p<0.001)$ RFs. Importantly, JTT in the Other RF resulted in a significantly higher JTT compared to the Object RF $(p<0.05)$ (Fig. 2). The Self condition is reported for completeness but not discussed further, firstly because not directly comparable, from a perceptual point of view, with the two allocentric conditions; secondly as beyond the focus of the current study.

The results of the first experiment confirm the hypothesis that using an allocentric but body-centered (Other) RF is different from using an allocentric but object-centered RF during extrapersonal space categorization. The adoption of an Other-centered RF indeed resulted in judging a greater portion of extrapersonal space as "Near" compared to adopting an Object-centered RF.

\section{Experiment 2}

According to the findings discussed above, the "Near" extrapersonal space is significantly greater in the Other condition vs the Object condition, suggesting that the effect is due to the particular nature of the allocentric RF: an agent's body versus an object. However, it is not clear whether the "Near space extension" is due to the processing of the human-like body shape per se or of a living human body. In fact, the avatar represents a living human agent potentially able to move towards the target, different from the static, inanimate object.

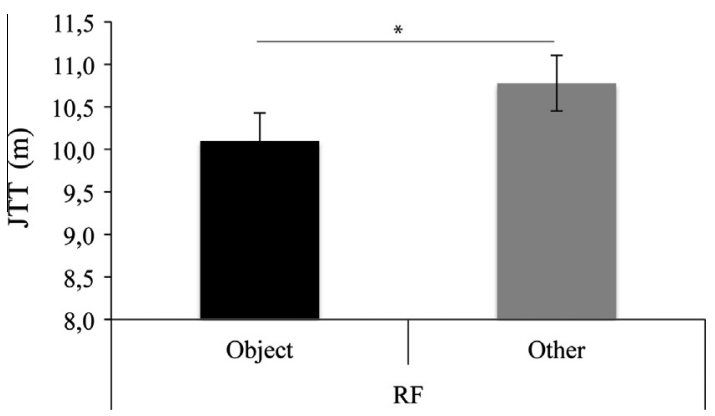

Fig. 2. Mean Judgment Transition Thresholds (JTTs) in Experiment 1.
In order to clarify this issue, we conducted a second VR experiment by introducing a non-living human-like agent (that is, a wooden dummy) as allocentric RF.

\subsection{Materials and methods}

Seventeen healthy subjects took part in this experiment (12 females; mean age 23 years; range 19-30). All subjects were right-handed, had normal or corrected-to-normal visual acuity and were naïve as to the purposes of the experiment. The study was approved by the Ethics Committee of the "G. d'Annunzio" University, Chieti, and conducted in accordance with the ethical standards of the 1964 Declaration of Helsinki.

In this experiment we replaced the Self condition with a new set of allocentric stimuli with a Dummy as RF (Fig. 1C). The experiment was thus composed of 3 RFs conditions (Object, Other, Dummy), for a total of 24 experimental series. The procedure was identical to the previous experiment.

\subsection{Results and discussion}

As in the previous experiment, we conducted a repeated measures ANOVA comparing the JTT in the three RFs conditions (Dummy, Other, Object). JTT analysis revealed a significant effect of RF-based distance perception $\left(F_{(2,32)}=\right.$ 9.88, $p<0.001, \eta^{2}=0.4$ ). Post-hoc tests (Newman Keuls) showed a significantly higher JTT with the Other RF (JTT $=8.85 \mathrm{~m}, \mathrm{SD}=3.7$ ) than with both the Dummy $\mathrm{RF}$ $(\mathrm{JTT}=7.64 \mathrm{~m}, \quad \mathrm{SD}=2.6 ; \quad p<0.001)$ and the Object $\mathrm{RF}(\mathrm{JTT}=7.36 \mathrm{~m}, \mathrm{SD}=2.53 ; p<0.001)$, which did not significantly differ ( $p=0.43$ ) (Fig. 3 ).

The human-like dummy, which is unable to move and walk towards the target, was thus processed more like a static object than as a living human agent. These data therefore suggest that the "Near space extension" that we observe when adopting an Other-centered RF is due to the biological nature of the human agent, equipped with motion potentialities.

\section{Experiment 3}

We have shown that a target object is judged as closer when the reference frame is a human agent compared with an object resembling (i.e., a wooden dummy) or not (i.e., an umbrella) a human body. We speculated that such "Near

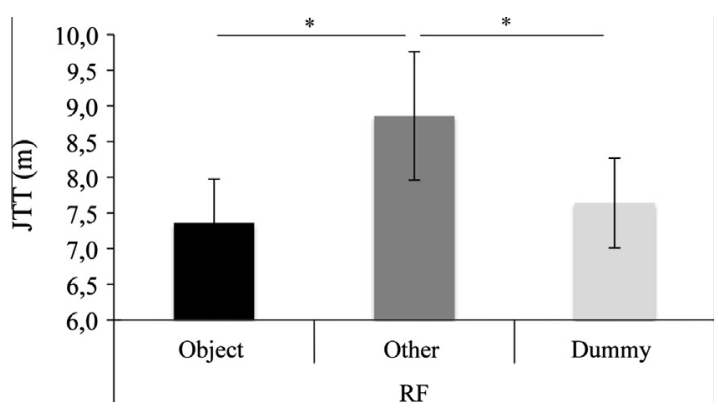

Fig. 3. Mean Judgment Transition Thresholds (JTTs) in Experiment 2. 
space extension" was due to the implicit processing of the motion potentialities intrinsic to the living human agent. However, the human-like dummy was not only unable to walk but also lacked of a basic perceptual apparatus (i.e. eyes). In order to rule out the possibility that the effects found in the previous experiments were due to visual perspective taking based on a simple line-of-sight (or visual accessibility) mechanism (Zacks \& Michelon, 2005) rather than to the agent's motor potentiality, we carried out a third VR experiment in which we compared a human agent free to move towards the target with a human agent whose motor potentialities were restricted. From the visibility point of view this condition is identical to the avatar without motor restriction, while the movement potentiality hypothesis predicts a difference in terms of extrapersonal space judged as near (i.e., lacking of a "Near space extension").

\subsection{Materials and methods}

Thirty healthy subjects took part in this experiment (25 females; mean age 21.5 years; range $20-21$ ). All but one subjects were right-handed, had normal or corrected-tonormal visual acuity, were naïve as to the purposes of the experiment. The study was approved by the Ethics Committee of the "G. d'Annunzio" University, Chieti, and conducted in accordance with the ethical standards of the 1964 Declaration of Helsinki.

In this experiment the Dummy condition was replaced with a new set of allocentric stimuli in which the avatar was tied to a pole with a rope (Fig. 1D). The experiment was thus composed of 3 RFs conditions (Object, Other, Tied-Other), for a total of 24 experimental series. The procedure was identical to the previous VR experiments.

\subsection{Results and discussion}

We conducted a repeated measures ANOVA comparing the JTT in the three RFs conditions (Object, Other, TiedOther). We obtained a marginally significant interaction $\left(F_{(2,58)}=2.81, p=0.056, \eta^{2}=0.9\right)$ and post hoc tests (Newman Keuls) showed a significantly higher JTT with the Other RF $(\mathrm{JTT}=10.98 \mathrm{~m}, \mathrm{SD}=3.39)$ than with the TiedOther RF (JTT $=10.64 \mathrm{~m}, \mathrm{SD}=3.44 ; p<0.04)$, which did not significantly differ from the Object $(J T T=10.69$,

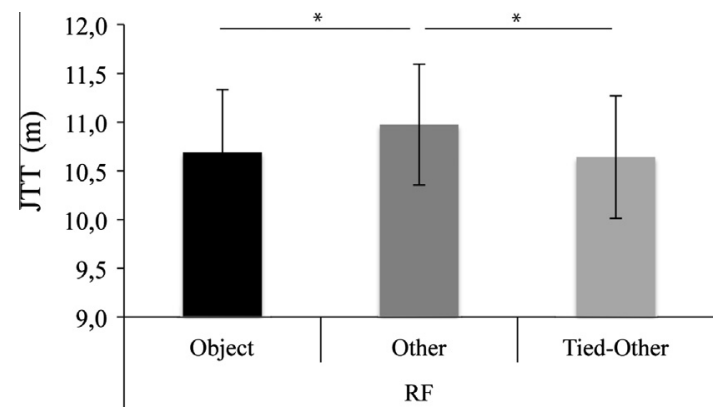

Fig. 4. Mean Judgment Transition Thresholds (JTTs) in Experiment 3. The difference between the Object RF and the Other RF was marginally significant $(p=0.056)$.
$\mathrm{SD}=3.53, p=0.75$ ) (Fig. 4). We can thus claim that the "Near space extension" observed when using a human body as RF is better accounted for by the RF's movement potentialities than by a basic line-of-sight perceptual mechanism.

\section{General discussion}

The general aim of the current study was to investigate the extrapersonal space categorization when using a human body as allocentric reference frame (RF).

In three virtual reality experiments we found that, when adopting another human body as RF the space we judge as "Near" is more extended compared to a condition in which we adopt an inanimate object as RF (Experiment 1). Moreover, such "Near space extension" is not present when we adopt as RF a dummy instead of a living human agent, showing that the human-like anatomical structure per se is not sufficient to induce the effect (Experiment 2 ). However, besides sharing the same general anatomical structure with the non-human agent, the human agent is additionally endowed with a perceptual system that might allow a judgment based on his line-of-sight (Zacks \& Michelon, 2005). To account for the "Near space extension" in terms of movement potentialities instead of a mere lineof-sight mechanism, a human agent inhibited in his motor resources has been compared with a human agent free to move, showing that the "Near" space was extended only when the human agent was free to move (Experiment 3).

Many theorists of perception have argued that the subjective experience of space, especially the perception of object distance, depends upon the movement possibilities of the agent (Declerck \& Gapenne, 2009). Over the last years, clear evidence for this claim has been found in the context of reaching in peripersonal space. It has been demonstrated that an object induces strong motor affordance when it is located within our reaching space (Costantini, Ambrosini, Tieri, Sinigaglia, \& Committeri, 2010) and we perceive an object as closer when it is reachable with a tool (Witt et al., 2005; Witt \& Proffitt, 2008).

Object affordances seem to be also influenced by considering other people's motor potentialities. Our motor system is, in fact, similarly triggered when the graspable object is located in the other's peripersonal space (Costantini, Committeri et al., 2011; Cardellicchio et al., 2013). The authors argued that such an effect is based on a shared mapping of one's own and others' arm reaching space. Moreover, we perceive an object as being closer when it is reachable by another individual (Costantini, Ambrosini et al., 2011; Bloesch, Davoli, Roth, Brockmole, \& Abrams, 2012). So, the other's body seems to be processed as a special stimulus within peripersonal space.

While most research has been carried out on the relationship of space and affordances in peripersonal space, much less is known about the influence of action potentialities on the perception of extrapersonal space. Noteworthy, a series of studies showed that extrapersonal space perception from an egocentric perspective is influenced by our bodily resources (Proffitt et al., 2003; Proffitt, 2006; Witt et al., 2004; Sugovic \& Witt, 2013). 
In our study we focused on the social (allocentric) counterpart of this embodied perception in extrapersonal space. We have demonstrated that the space in a distance judgment is experienced in a particular way when the RF is the body of another person. Given that the other individual in the scene constitutes the spatial reference for our judgment, his/her intrinsic action opportunities influence our space categorization. We seem to consider not only our own but also another person's motor resources when judging the space around us, showing a shared categorization of the extrapersonal space. Therefore "perception could scale the geometry of spatial layout to the economy of possible human action" (Proffitt, 2006), including all human beings present in the scene. We propose that the distance between a human being and a target could be processed as less expanded than the distance between two objects, as we implicitly consider the other's abilities to reach it, filtering the extrapersonal space from the other's legs.

Nevertheless, we cannot know the process behind the movement elaboration of the "other" body in spatial categorization. One possible mechanism behind this elaboration is an automatic perspective taking, which has been demonstrated in different kind of visuospatial tasks (e.g., Tversky \& Hard, 2009; Kessler \& Rutherford, 2010; Samson et al., 2010; Surtees \& Apperly, 2012). However, our task was not designed to test perspective taking and therefore does not allow to disentangle whether it is present and in which form/level.

What our data suggest is that using as RF for space categorization a virtual body being able to move triggers a representation of the action afforded by the environment. Such action representation in the Other-based RF could be based on the internal simulation process of the intended/potential action, i.e. walking towards the target, a cognitive process well described by the neuro-cognitive model of space categorization and selection for action of Coello and Delevoye-Turrell (2007). The key aspect of the model for the present investigation is that space categorization is directly affected by the whole simulation process, which not only shapes and prepares the motor system for the consequences of motor execution, but also provides the self with information on the feasibility of potential actions. Our data suggest that such representation of the "functional body" with its potential actions arise not only in relation to the observer's own body but also to the body of other individuals located in an extended space. The pre-reflective internal simulation of the action could be a process common both to our implicit action planning and to inferred action planning of others.

However, we are not able to exclude that the observed "Near space extension" is due to an abstract, disembodied processing of RF movement potentialities. In principle, the human body could influence our space perception as a "tool" with motion opportunities and not necessarily because it is a human RF. To explore this possibility, further investigations are required, focusing on RFs without human resemblance but able to move.

The proposed 'social' scaling of extrapersonal space could have an evolutionary basis. The other human being, in fact, could represent a potential competitor in the environment, so the underestimation of the distance when adopting his/her body as RF could lead us to spend more energy to get the target, e.g. some food. From this perspective, Hemmi and Zeil (2003) showed that arthropods can judge how close are other arthropods to their burrow, and the likelihood of rushing back to defend their burrows increases the smaller is the distance between the competitor and the burrow. On the basis of our results, we speculate that also the human being could be an able detector of inter-object distance, considering the relevant motion possibilities of potential competitors in the environment. Future studies could explore this hypothetical evolutionary basis of "social" scaling, by using a competitive allocentric $\mathrm{RF}$ and a target to compete for.

In conclusion, this research demonstrated that during allocentric distance judgments within the extrapersonal space we implicitly process the movement potentialities of the reference frame. In particular, the Other-based reference frame represents a special kind of "allocentric" spatial reference for which a greater portion of space is categorized as "near" or accessible compared to a static inanimate object or to non-biological agents. In Other-based coordinates, extrapersonal space should be considered as a "ready to walk" space, where distances are mentally "travelled" and not simply observed.

\section{Acknowledgement}

The work was supported by University G. d'Annunzio funds to GC.

\section{References}

Becchio, C., Del Giudice, M., Latini-Corazzini, L., \& Pia, L. (2011). In your place: Neuropsychological evidence for alterocentric remapping in embodied perspective taking. Social Cognitive and Affective Neuroscience, 8, 165-170.

Bloesch, E. K., Davoli, C. C., Roth, N., Brockmole, J. R., \& Abrams, R. A. (2012). Watch this! Observed tool use affects perceived distance. Psychonomic Bulletin and Review, 19, 177-183.

Cardellicchio, P., Sinigaglia, C., \& Costantini, M. (2013). Grasping affordances with the other's hand: A TMS study. Social Cognitive and Affective Neuroscience, 8(4), 455-459.

Coello, Y., \& Delevoye-Turrell, Y. (2007). Embodiment, spatial categorization, action. Consciousness and Cognition, 16, 667-683.

Costantini, M., Ambrosini, E., Sinigaglia, C., \& Gallese, V. (2011). Tool-use observation makes far objects ready-to-hand. Neuropsychologia, 49, 2658-2663.

Costantini, M., Ambrosini, E., Tieri, G., Sinigaglia, C., \& Committeri, G. (2010). Where does an object trigger an action? An investigation about the affordances in the space. Experimental Brain Research, 207, 95-103.

Costantini, M., Committeri, G., \& Sinigaglia, C. (2011). Ready both to your and to my hands: Mapping the action space of others. PLOS ONE, 6, e17923.

Cutting, J. E., \& Vishton, P. M. (1995). Perceiving layout: The integration, relative dominance, and contextual use of different information about depth. In W. Epstein \& S. Rogers (Eds.), Handbook of perception and cognition: Perception of space and motion (pp. 69-117). New York: Academic Press.

Declerck, G., \& Gapenne, O. (2009). Actuality and possibility: On the complementarity of two registers in the bodily constitution of experience. Phenomenology and Cognitive Science, 8, 285-305.

Grusser, O.-J. (1983). Multimodal structure of the extrapersonal space. In A. Hein \& M. Jeannerod (Eds.), Spatially oriented behavior (pp. 327-352). New York: Springer-Verlag.

Hemmi, J. M., \& Zeil, J. (2003). Robust judgement of inter-object distance by an arthropod. Nature, 421, 160-163.

Kessler, K., \& Rutherford, H. (2010). The two forms of visuo-spatial perspective taking are differently embodied and subserve different spatial prepositions. Frontiers in Psychology, 1, 213. 
Previc, F. (1998). The neuropsychology of 3-D space. Psychological Bulletin, 124(2), 123-164.

Proffitt, D. R. (2006). Embodied perception and the economy of action. Perspectives on Psychological Science, 1, 110-122.

Proffitt, D. R., Stefanucci, J., Banton, T., \& Epstein, W. (2003). The role of effort in distance perception. Psychological Science, 14, 106-113.

Rizzolatti, G., Fadiga, L., Fogassi, L., \& Gallese, V. (1997). The space around us. Science, 277, 190-191.

Samson, D., Apperly, I., Braithwaite, J., Andrews, B., \& Scott, S. (2010). Seeing it their way: Evidence for rapid and involuntary computation of what other people see. Journal of Experimental Psychology: Human Perception and Performance, 36, 1255-1266.

Sugovic, M., \& Witt, J. K. (2013). An older view on distance perception: Older adults perceive walkable extents as farther. Experimental Brain Research, 226, 383-391.
Surtees, A. D., \& Apperly, I. A. (2012). Egocentrism and automatic perspective taking in children and adults. Child Development, 83(2), 452-460.

Tversky, B., \& Hard, M. B. (2009). Embodied and disembodied cognition: Spatial perspective taking. Cognition, 110, 124-129.

Witt, J. K., \& Proffitt, D. R. (2008). Action-specific influences on Distance perception: A role for motor simulation. Journal of experimental psychology: Human perception and Performance, 34, 1479-1492.

Witt, J. K., Proffitt, D. R., \& Epstein, W. (2004). Perceiving distance: A role of effort and intent. Perception, 33, 577-590.

Witt, J. K., Proffitt, D. R., \& Epstein, W. (2005). Tool use affects perceived distance, but only you intend to use it. Journal of experimental psychology: Human perception and Performance, 31, 880-888.

Zacks, J. M., \& Michelon, P. (2005). Transformations of visuospatial images. Behavioral and Cognitive Neuroscience Reviews, 4, 96-118. 